\title{
Nature of $e_{g}$ Electron Order in $\mathrm{La}_{1-x} \mathrm{Sr}_{1+x} \mathrm{MnO}_{4}{ }^{*}$
}

\author{
S. Larochelle, ${ }^{1}$ A. Mehta, ${ }^{2}$ N. Kaneko, ${ }^{2}$ P. K. Mang, ${ }^{3}$ A. F. Panchula, ${ }^{3}$ L. Zhou, ${ }^{4}$ J. Arthur ${ }^{2}$ \\ and M. Greven ${ }^{2,3}$ \\ ${ }^{1}$ Department of Physics, Stanford University, Stanford, CA 94305 \\ ${ }^{2}$ Stanford Synchrotron Radiation Laboratory, Stanford Linear Accelerator Center, \\ Stanford, CA 94309 \\ ${ }^{3}$ Department of Applied Physics, Stanford University, Stanford, CA 94305 \\ ${ }^{4}$ T. H. Geballe Laboratory for Advanced Materials, Stanford University, \\ Stanford, CA 94305
}

\begin{abstract}
Synchrotron x-ray scattering measurements of the low-temperature structure of the single-layer manganese oxide $\mathrm{La}_{1-x} \mathrm{Sr}_{1+x} \mathrm{MnO}_{4}$ over the doping range $0.33 \leq$ $x \leq 0.67$, indicate the existence of three distinct regions: a disordered phase $(x<0.4)$, a charge-ordered phase $(x \geq 0.5)$, and a mixed phase $(0.4 \leq x<$ $0.5)$. For $x>0.5$, the modulation vector associated with the charge order is incommensurate with the lattice and depends linearly on the concentration of $e_{g}$ electrons. The primary superlattice reflections are strongly suppressed along the modulation direction and the higher harmonics are weak, implying the existence of a largely transverse and nearly sinusoidal structural distortion, consistent with a charge density wave of the $e_{g}$ electrons.
\end{abstract}

Submitted to Physical Review Letter

*Work supported by Department of Energy contract DE-AC03-76SF00515. 
Perovskite-derived transition metal oxides have attracted much attention over the past decade because of their unusual electronic properties. Strong electron correlations give rise to such phenomena as high-temperature superconductivity in layered cuprates and stripe-like order in layered cuprates and nickelates. In the case of the manganites, an additional strong interaction among charge and lattice degrees of freedom leads to a very rich electronic phase diagram in which crystallographic and magnetic structures as well as transport properties are intimately related. The competition among various phases has been closely associated [1] with the colossal magnetoresistance (CMR) observed in these materials. For example, the perovskite $\mathrm{La}_{1-x} \mathrm{Ca}_{x} \mathrm{MnO}_{3}$ exhibits two low-temperature phases at doping $x \sim 0.5$, one a ferromagnetic metal and the other an antiferromagnetic insulator [2,3]. The insulating phase at the doping level $x=0.5$, where there is on average half an $e_{g}$ electron per manganese atom, is characterized by a complex checkerboard arrangement of ordered $e_{g}$ electrons, orbitals and spins (CE-type antiferromagnetic order) [4-6]. The ordered phase of $\mathrm{La}_{1-x} \mathrm{Ca}_{x} \mathrm{MnO}_{3}$ extends to rather high doping where the average number $n_{e}=1-x$ of $e_{g}$ electrons decreases markedly and, consequently, the low-temperature unit cell becomes very large $[7,8]$.

CMR has been observed in the perovskite and double-layer manganites, but not in the single-layer system $[9,10]$, the most nearly two-dimensional member of this series. Nevertheless, there are signs that the physics of $\mathrm{La}_{1-x} \mathrm{Sr}_{1+x} \mathrm{MnO}_{4}$ is similar to that of the perovskite manganites. For example, CE-type antiferromagnetic order has been reported for $\mathrm{La}_{0.50} \mathrm{Sr}_{1.50} \mathrm{MnO}_{4}$ [11]. Moreover, the low-temperature phase is sensitive to magnetic fields [12], and it extends to high doping (low $e_{g}$ electron concentration) [10]. A systematic study of the low-temperature structural phases of $\mathrm{La}_{1-x} \mathrm{Sr}_{1+x} \mathrm{MnO}_{4}$ should provide valuable insights into the effect of dimensionality on the properties of the manganites. Such a study may also contribute to a deeper understanding of the single-layer transition metal oxides.

In this Letter, we report a non-resonant x-ray scattering study of $\mathrm{La}_{1-x} \mathrm{Sr}_{1+x} \mathrm{MnO}_{4}$ $(0.33 \leq x \leq 0.67)$. We first consider the low-temperature structure of $\mathrm{La}_{0.50} \mathrm{Sr}_{1.50} \mathrm{MnO}_{4}$. Our data provide a more complete picture than previous neutron [11] and x-ray [13] scattering experiments. We then extend our investigation to study the effects of varying the 
$e_{g}$ electron concentration in the $\mathrm{MnO}_{2}$ layers. We find three distinct regions: disordered $(x<0.4)$, mixed-phase $(0.4 \leq x<0.5)$, and charge-ordered $(x \geq 0.5)$. Above $x=0.5$, the ordering of $e_{g}$ electrons results in a structural distortion whose modulation period only depends on $n_{e}$. The distortion is largely transverse and nearly sinusoidal, consistent with charge-density wave order of the $e_{g}$ electrons.

Samples of $\mathrm{La}_{1-x} \mathrm{Sr}_{1+x} \mathrm{MnO}_{4}$ were prepared from stoichiometric amounts of $\mathrm{La}_{2} \mathrm{O}_{3}, \mathrm{MnO}_{2}$ and $\mathrm{SrCO}_{3}$ powders. The mixtures were calcinated three times for twelve hours between $1300^{\circ} \mathrm{C}$ and $1360^{\circ} \mathrm{C}$. The calcinated powders were then pressed into rods of $5 \mathrm{~mm}$ diameter and sintered at $1600^{\circ} \mathrm{C}$ for twelve hours. Single crystals were grown by the floating-zone method at a rate of $6 \mathrm{~mm} /$ hour in an oxygen atmosphere of 5bars. Crystal pieces of dimensions $2 \times 4 \times 1 m^{3}$ were cut from the grown boules and were mounted inside a closedcycle refrigerator. The mosaic widths of the crystals used in this study were $0.02-0.06^{\circ}$ (FWHM). The data were collected using a four-circle diffractometer at beamline 7-2 of the Stanford Synchrotron Radiation Laboratory. A monochromatic x-ray beam was obtained from the wiggler spectrum via a Si (111) double-crystal monochromator. An x-ray energy of $14 \mathrm{keV}$ was selected to provide a penetration depth of about $40 \mu \mathrm{m}$ in order to study the bulk structure.

The low-temperature phase of $\mathrm{La}_{0.50} \mathrm{Sr}_{1.50} \mathrm{MnO}_{4}$ was probed by scanning along the highsymmetry directions in reciprocal space. Two such scans are presented in Fig. 1. The essential features of the ordered structure do not change below the charge-ordering temperature $T_{C O} \approx 240 \mathrm{~K}$. In addition to the peaks present in the high-temperature (I4/mmm) structural phase (henceforth referred to as high-symmetry peaks), peaks with wavevectors $\left(\frac{1}{4}, \frac{1}{4}, 0\right)_{t}$ and $\left(\frac{1}{2}, \frac{1}{2}, 0\right)_{t}$ were found (the subscripts $t$ and $o$ indicate, respectively, the tetragonal and orthorhombic unit cells). The $\left(\frac{1}{4}, \frac{1}{4}, 0\right)_{t}$ peaks ( $h$-odd peaks in the orthorhombic notation used in Fig. 1) suffer a near extinction along $(h, h, 0)_{t}\left((h, 0,0)_{o}\right.$ in orthorhombic notation). The presence of a quarter-wave modulation has been reported in electron diffraction measurements $[9,10]$. A neutron diffraction study found only a half-wave modulation and concluded that the quarter-wave modulation seen in the electron diffraction comes from 
a surface phase [11]. The measurements reported here were performed at relatively high $\mathrm{x}$-ray energy $(14 \mathrm{keV})$ to achieve a bulk penetration depth and were reproduced on samples from three different growths. Moreover, preliminary neutron scattering measurements have confirmed the presence of the quarter-wave peaks in the bulk.

Since some of the quarter-wave peaks are very intense (up to $\sim 1 \%$ of the intensity of the high-symmetry peaks, see Fig. 1) and are visible in neutron as well as in non-resonant x-ray scattering, the predominant contribution to the intensity of these peaks must come from a structural distortion and not from charge or orbital ordering on the Mn sites. We note that the origin of the resonant scattering signal observed by Murakami et al. [13] for $x=\frac{1}{2}$ is ambiguous since either the structural distortion reported here or direct charge and orbital ordering could be the source of such scattering $[14,15]$.

The existence of a quarter-modulation wavevector along $(h, h, 0)_{t}$ seems to imply that the $a$ and $b$ axes of the low-temperature phase are quadrupled. However, a smaller (half as large) orthorhombic unit cell, rotated by $45^{\circ}$ relative to the high-symmetry (I4/mmm) cell, and its $90^{\circ}$ twin, are adequate to index all the observed reflections: $a_{o}=2 \sqrt{2} a_{t}$, $b_{o}=\sqrt{2} a_{t}$, and $c_{o}=c_{t}$ ( $a_{t}$ and $c_{t}$ are the tetragonal lattice constants). We did not notice any superstructure ordering along the $c$ axis, the stacking direction of the $\mathrm{MnO}_{2}$ layers, or any difference between $a_{o}$ and $2 b_{o}$ (no splitting of the high-symmetry peaks within the experimental resolution). This orthorhombic unit cell is in agreement with the one used to describe the charge-ordered phases of the perovskite $\mathrm{La}_{0.50} \mathrm{Ca}_{0.50} \mathrm{MnO}_{3}$ [16] and the bi-layer material $\mathrm{LaSr}_{2} \mathrm{Mn}_{2} \mathrm{O}_{7}$ [17]. However, it is very different from the unit cell proposed by Sternlieb et al. for $\mathrm{La}_{0.50} \mathrm{Sr}_{1.50} \mathrm{MnO}_{4}[11]$.

After an extensive survey of reciprocal space, we conclude that the low-temperature structural symmetry belongs to extinction class $\mathrm{A}---$. This extinction class contains three space groups: Ammm and its two subgroups, Am2m and A222. A common characteristic of these structures is that they contain three unique Mn sites in a $1: 1: 2$ ratio and that equivalent Mn sites are located as far apart from each other as possible. The octahedral oxygen environment around each crystallographically unique Mn site is distinct and hence it 
is not unreasonable to assume that the three Mn are electronically inequivalent. The $h$-odd reflections are heavily suppressed along $(h, 0,0)_{o}$, implying that the structural distortions are not isotropic, but mostly orthogonal to the modulation direction $h$. Such a structural distortion is probably a shear-type distortion of the Mn-O octahedra (perhaps a Jahn-Teller distortion) similar to the one proposed for $\mathrm{La}_{0.50} \mathrm{Ca}_{0.50} \mathrm{MnO}_{3}$ [16] and $\mathrm{LaSr}_{2} \mathrm{Mn}_{2} \mathrm{O}_{7}$ [17], rather than a breathing-type distortion of the oxygen octahedra as proposed in Ref. [11]. To fully characterize the distortion, comprehensive high-resolution crystallography of the low-temperature phase will be necessary.

The nature of the distortion can be more clearly identified by studying the effects of varying the $e_{g}$ electron concentration $n_{e}=1-x$. We observed superlattice peaks in all samples with $x>0.40$, up to $x=0.67$, the highest value investigated. For $x>0.50$, the superlattice modulation vector changes linearly with $n_{e}$, as shown in Fig. 3(a). For $x=0.50$, the superlattice modulation doubles the high-temperature structure (along the base diagonal), and for $x=0.67$, it triples it. This linear dependence of the wave vector is similar to that observed in $\mathrm{La}_{1-x} \mathrm{Ca}_{x} \mathrm{MnO}_{3}$ for $x>0.5$ [7] and, in particular, at $x=\frac{2}{3}$ [8,18-20]. While at $x=\frac{1}{2}$ and $x=\frac{2}{3}$ commensurate wavevectors are observed, the ordering is best understood, at all doping levels $\frac{1}{2} \leq x \leq \frac{2}{3}$, as a modulation whose period only is a function of $n_{e}$. Because the superlattice modulation is directly correlated with $n_{e}$, it is likely that the structural phase transition is driven by the $e_{g}$ electrons.

Second and third diffraction harmonics, much weaker than the primary (labeled as 2, 3 and 1 in Fig. 2), are visible at $( \pm 2 \epsilon, 0,0)_{o}$ and $( \pm 3 \epsilon, 0,0)_{o}$. Weak scattering at higher diffraction harmonics may, in principle, result from a pure sine wave modulation [21]. However, even if the presence of the higher harmonics is an indication of a non-sinusoidal component of the distortion, the weakness of the higher harmonic intensities suggests that the structural distortion is essentially sinusoidal. The widths of the superlattice peaks are comparable to those of the high-symmetry peaks which implies that the low-temperature phase exhibits long-range order.

The nearly sinusoidal structural distortion, together with the linear variation of the 
modulation wavevector with doping, precludes any model in which the $e_{g}$ electron ordering is too closely linked to the underlying cationic lattice, such as the bi-stripe model of Ref. [8] or the discommensurate-stripe model proposed for the single-layer nickelates [22]. A better description is given by a nearly sinusoidal structural distortion, probably associated with a charge density wave. The variation of the charge density, with equivalent Mn sites located as far apart as possible, is similar to the "Wigner-crystal" (or stripe-like) arrangement of the $e_{g}$ electrons proposed for $\mathrm{La}_{0.333} \mathrm{Ca}_{0.667} \mathrm{MnO}_{3}$ [18].

A $2^{\circ}$ rotation of the modulation direction with respect to the high-temperature lattice was found in electron diffraction studies of $\mathrm{La}_{0.33} \mathrm{Ca}_{0.67} \mathrm{MnO}_{3}$ [20]. We did not observe any rotation of the structural modulation in $\mathrm{La}_{1-x} \mathrm{Sr}_{1+x} \mathrm{MnO}_{4}$ up to $x=0.67$, the highest doping level of our study. Figure 3(b) shows the scattering intensity for $x=0.525$ around two second harmonic points in the $(h, k, 0)_{o}$ plane. Within the experimental error, the two reflections have the same $k$ value and are not broadened.

For $x<0.40$, there is no superlattice structure at low temperature. Rather, we observe very weak diffuse scattering centered around specific locations of reciprocal space. We have observed similar diffuse scattering for $x \geq 0.50$ above the charge-ordering temperature. The diffuse scattering pattern in the single-layer manganite is complex, and we are currently in the process of carefully documenting it. However, we believe that some of the diffuse intensity may arise from short-range polaron-polaron correlations similar to those reported for the paramagnetic (insulating) phase of $\mathrm{La}_{0.7} \mathrm{Ca}_{0.3} \mathrm{MnO}_{3}$ [23]. Thus, there is no longrange distortion of the high-temperature struture for $x<0.40$, and the $e_{g}$ electrons are not ordered. Unlike the CMR manganites, $\mathrm{La}_{1-x} \mathrm{Sr}_{1+x} \mathrm{MnO}_{4}$ remains insulating in this region of doping $[9,10]$.

For $0.40<x<0.50$, the modulation vector remains the same as for $x=0.50$. However, as can be seen from Fig. 4, the intensity of the superlattice peak increases as $x$ increases toward $x=0.50$. The simplest explanation for this behavior is that the material separates into charge-ordered regions of $0.5 e_{g}$ electrons per Mn site and disordered regions of approximately $0.6 e_{g}$ electrons per Mn site. Additional support for this interpretation comes 
from the widths of the diffraction peaks. For $x \geq 0.45$, the widths of the superlattice peaks are nearly constant and comparable to the widths of the high-symmetry peaks. However, for $x<0.45$, the superlattice peak widths are ten to twenty times broader and the peak intensities are much reduced, implying that the remaining ordered domains are of relatively small size.

In conclusion, we have used synchrotron x-ray scattering to investigate the nature of the low-temperature structural distortion of $\mathrm{La}_{1-x} \mathrm{Sr}_{1+x} \mathrm{MnO}_{4}$ and its dependence on the $e_{g}$ electron concentration $n_{e}=1-x$. At $x=0.50$, our measurements indicate a sheartype structural distortion of the Mn-O octahedra. In light of this observation, we suggest a reexamination of previous resonance x-ray scattering results [13]. As a function of doping $x$, we found three distinct regions. For $x \geq 0.50$, the largely transverse structural distortion exhibits long-range order and the modulation vector of the distortion varies linearly with $n_{e}: \epsilon=2 n_{e}$. This distortion, which is similar to that found for $x=2 / 3$ in $\mathrm{La}_{1-x} \mathrm{Ca}_{x} \mathrm{MnO}_{3}$ $[18,20]$, appears to be associated with a nearly sinusoidal charge density wave. Samples with $x<0.40$ exhibit no peaks corresponding to long-range superstructural order, but only diffuse scattering peaks, perhaps due to short-range polaron-polaron correlations. The intermediate region, $0.40 \leq x<0.50$, is best understood as a mixture of the ordered and the disordered phases. This phase diagram is reminiscent of that of the CMR perovskite $\mathrm{La}_{1-x} \mathrm{Ca}_{x} \mathrm{MnO}_{3}$. The structure of the ordered phase appears to primarily depend on $n_{e}$, and to be rather insensitive to the dimensionality of the lattice. Further, both the single-layer system and the CMR perovskites phase separate for $n_{e}>0.5$. Nevertheless, for $n_{e}>0.5$, their macroscopic low-temperature transport and magnetic properties differ dramatically.

We would like to acknowledge helpful discussions with B. W. Batterman, F. Bridges, E. Dagatto, S. Ishihara, S. A. Kivelson, J. W. Lynn and S. Maekawa. SSRL is operated for the U.S. Department of Energy, Office of Basic Energy Sciences by Stanford University. This work was supported by the U.S. Department of Energy under Contracts Nos. DEFG03-99ER45773-A001 and DE-AC03-76SF00515. M.G. is also supported by the A.P. Sloan Foundation. 


\section{REFERENCES}

[1] A. Moreo, S. Yunoki, and E. Dagotto, Science 283, 2034 (1999).

[2] S. Mori, C. H. Chen, and S.-W. Cheong, Phys. Rev. Lett. 81, 3972 (1998).

[3] Q. Huang et al., Phys. Rev. B 61, 8895 (2000).

[4] E. O. Wollan and W. C. Koehler, Phys. Rev. 100, 545 (1955).

[5] J. B. Goodenough, Phys. Rev. 100, 564 (1955).

[6] Y. Tokura and N. Nagaosa, Science 288, 462 (2000).

[7] C. H. Chen, S.-W. Cheong, and H. Y. Hwang, J. Appl. Phys. 81, 4326 (1997).

[8] S. Mori, C. H. Chen, and S.-W. Cheong, Nature (London) 392, 473 (1998).

[9] Y. Moritomo et al., Phys. Rev. B 51, 3297 (1995).

[10] W. Bao, C. H. Chen, S. A. Carter, and S.-W. Cheong, Solid State Commun. 98, 55 (1996).

[11] B. J. Sternlieb et al., Phys. Rev. Lett. 76, 2169 (1996).

[12] M. Tokunaga, N. Miura, Y. Moritomo, and Y. Tokura, Phys. Rev. B 59, 11151 (1999).

[13] Y. Murakami et al., Phys. Rev. Lett. 80, 1932 (1998).

[14] M. Benfatto, Y. Joly, and C. R. Natoli, Phys. Rev. Lett. 83, 636 (1999).

[15] I. S. Elfimov, V. I. Anisimov, and G. A. Sawatzky, Phys. Rev. Lett. 82, 4264 (1999).

[16] P. G. Radaelli, D. E. Cox, M. Marezio, and S.-W. Cheong, Phys. Rev. B 55, 3015 (1997).

[17] D. N. Argyriou et al., Phys. Rev. B 61, 15269 (2000).

[18] P. G. Radaelli et al., Phys. Rev. B 59, 14440 (1999). 
[19] M. T. Fernández-Díaz, J. L. Martínez, J. M. Alonso, and E. Herrero, Phys. Rev. B 59, 1277 (1999).

[20] R. Wang, J. Gui, Y. Zhu, and A. R. Moodenbaugh, Phys. Rev. B 61, 11946 (2000).

[21] J. D. Axe, Phys. Rev. B 21, 4181 (1980).

[22] H. Yoshizawa et al., Phys. Rev. B 61, R854 (2000).

[23] C. P. Adams et al., Phys. Rev. Lett. 85, 3954 (2000). 


\section{FIGURES}

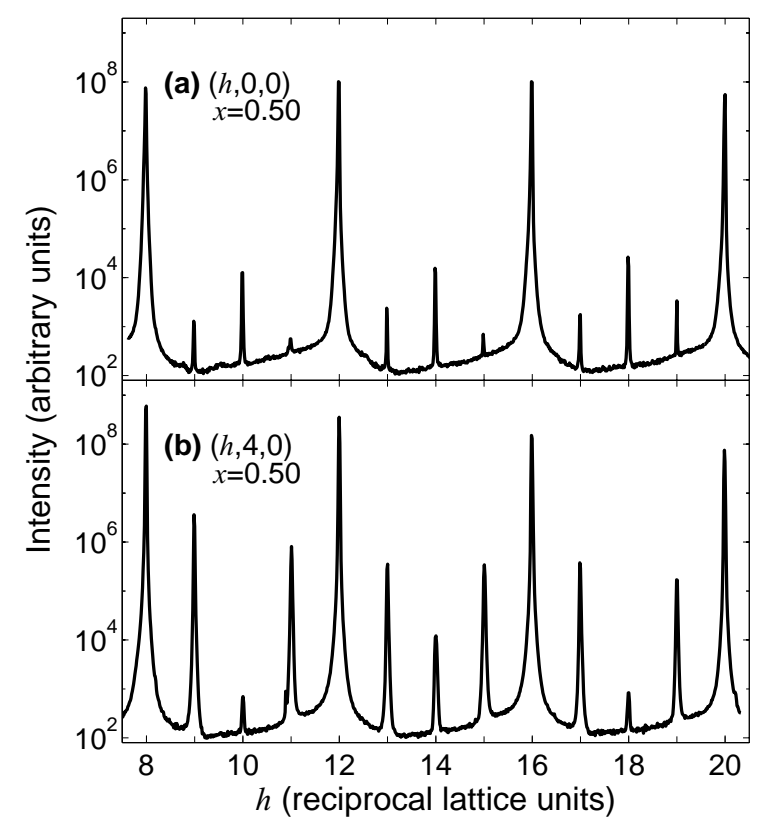

FIG. 1. X-ray diffraction scans of $\mathrm{La}_{0.50} \mathrm{Sr}_{1.50} \mathrm{MnO}_{4}$ in the low-temperature phase $(\mathrm{T}=7 \mathrm{~K})$ along (a) $(h, 0,0)_{o}$ and (b) $(h, 4,0)_{o}$ ("o" indicates the orthorhombic unit cell). The peaks at $h=8,12,16$ and 20 are present in the high-temperature phase. The superlattice peaks at odd-integer values of $h$ are strongly suppressed along $(h, 0,0)_{o}$. Note the logarithmic intensity scale. 


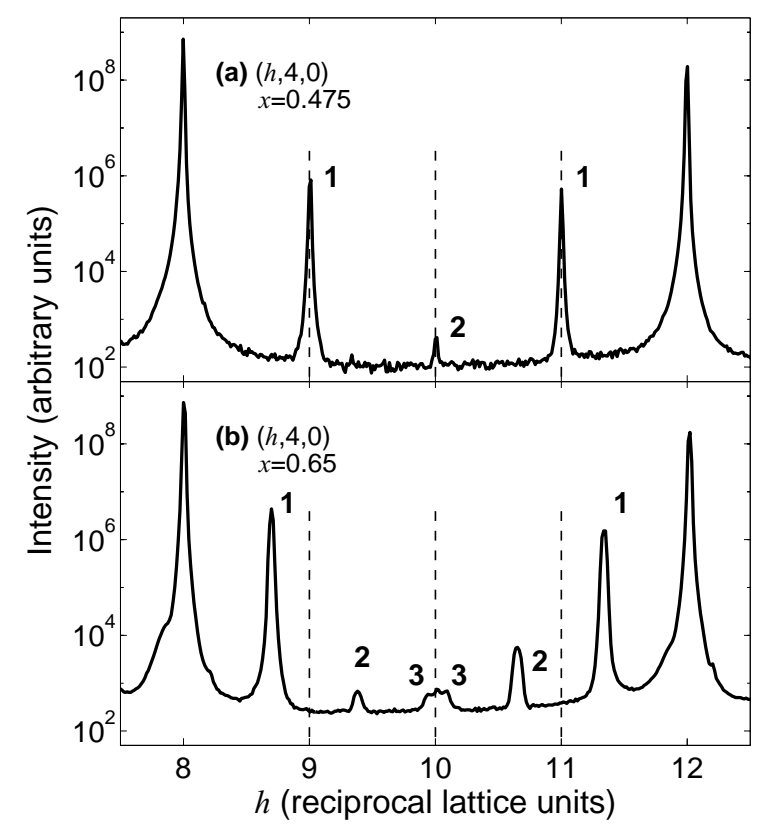

FIG. 2. X-ray diffraction scans of $\mathrm{La}_{1-x} \mathrm{Sr}_{1+x} \mathrm{MnO}_{4}$ in the low-temperature phase $(\mathrm{T}=7 \mathrm{~K})$ along $(h, 4,0)_{o}$ for (a) $x=0.475$ and (b) $x=0.65$. The vertical dashed lines indicate the commensurate positions. 1, 2 and 3 label, respectively, the first, second and third harmonics of the low-temperature distortion. Note the logarithmic intensity scale. 

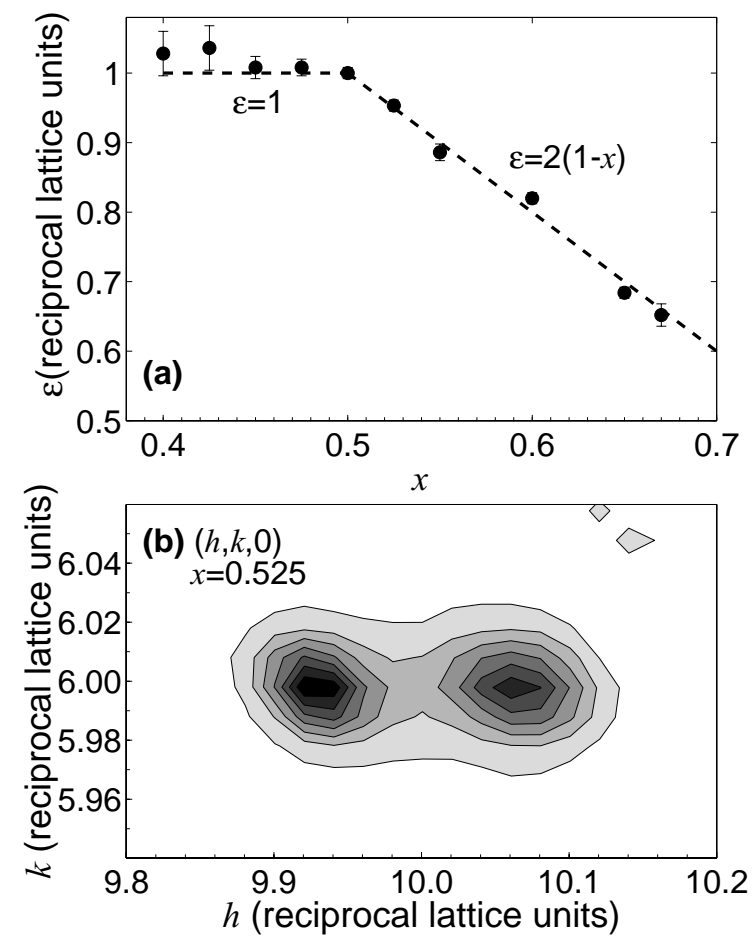

FIG. 3. (a) Superlattice wave vector $( \pm \epsilon, 0,0)_{o}$ as a function of $x$ for $\mathrm{La}_{1-x} \mathrm{Sr}_{1+x} \mathrm{MnO}_{4}$. The dashed line for $x>0.5$ is $\epsilon=2(1-x)=2 n_{e}$. (b) Linear-scale contour map (10\% contours) of the scattering intensity around $(10,6,0)_{o}$ for $x=0.525$. 


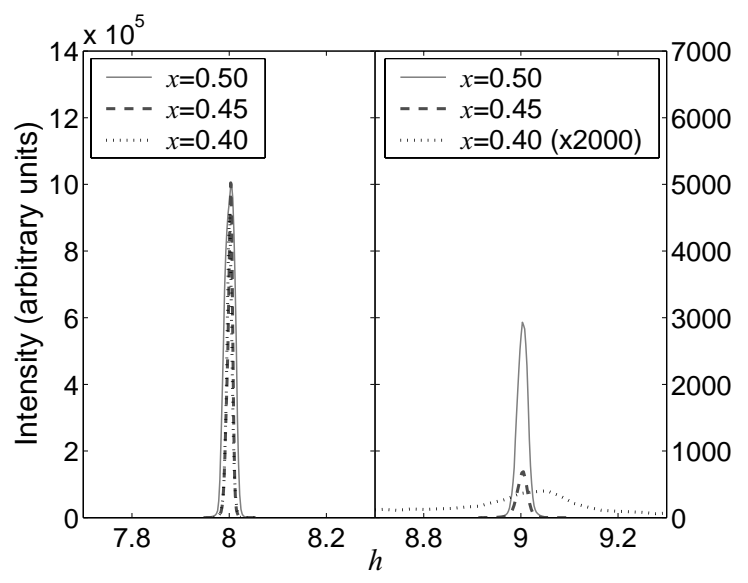

FIG. 4. Scans between the $(8,4,0)_{o}$ and the $(9,4,0)_{o}$ reflections at $x=0.40,0.45$ and 0.50 $(\mathrm{T}=100 \mathrm{~K})$. The $(8,4,0)_{o}$ peak intensities are normalized to $10^{6}$. Below $x=0.50$, the $(9,4,0)_{o}$ superlattice peak intensity decreases considerably with decreasing $x$. The $x=0.40$ peak is noticeably broadened and its intensity is $\sim 10^{4}$ weaker than the $x=0.5$ peak. 